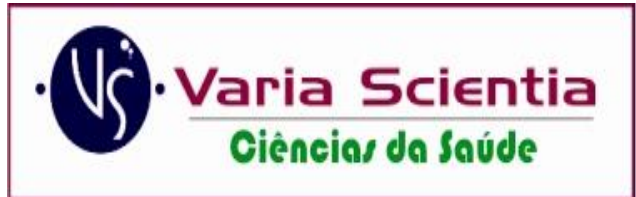

e-ISSN 2446-8118

\title{
A INFLUÊNCIA DO TABAGISMO SOBRE A MECÂNICA RESPIRATÓRIA DE ADULTOS JOVENS
}

\author{
THE INFLUENCE OF SMOKING ON THE RESPIRATORY MECHANICS OF YOUNG \\ ADULTS
LA INFLUENCIA DE FUMAR EN LA MECÁNICA RESPIRATORIA DE ADULTOS JÓVENES

Letícia Sousa Silva ${ }^{1}$

David Halen Araújo Pinheiro ${ }^{2}$

Daniele Naiara Araujo Pinheiro ${ }^{3}$

Ana Flávia Machado de Carvalho ${ }^{4}$

RESUMO: Objetivo: avaliar os efeitos do tabagismo sobre a mecânica respiratória em adultos jovens. Métodos: esse estudo é delineado como pesquisa de campo, de abordagem quantitativa, descritiva e de natureza comparativa. A amostra do estudo foi composta por 18 participantes, escolhidos de acordo com os critérios pré-estabelecidos. Para coleta de dados, utilizou-se uma ficha de avaliação elaborada pelos pesquisadores, um manovacuômetro analógico para mensurar a força dos músculos respiratórios por meio da PImax e da PEmax e um Peak- Flow para medir o pico de fluxo expiratório. Resultados: a maioria dos participantes $(n=12) 66,7 \%$ eram do gênero masculino e $(n=6) 33,3 \%$ do gênero feminino. Dos $(n=10) 55,6 \%$ tabagistas $(n=6) 60 \%$ haviam iniciado fumo antes dos 18 anos. Segundo a escala de dependência de nicotina 30\% do grupo apresentou grau elevado $(n=3)$. Houve diferença estatisticamente significativa entre média de altura entre jovens com nível 1 em relação aqueles com nível 2 de dependência de nicotina $(1,76$ versus 1,$67 ; \mathrm{p}=0,01)$. Conclusão: $O$ hábito apresenta efeitos deletérios, desenvolvendo uma dependência química e alterando a mecânica respiratória de adultos jovens.

DESCRITORES: Jovens adultos; Tabagismo; Mecânica respiratória; Fisioterapia.

\begin{abstract}
Objective: to evaluate the effects of smoking on respiratory mechanics in young adults. Methods: this study is designed as a field research, with a quantitative, descriptive and comparative approach. The study sample consisted of 18 participants, chosen according to preestablished criteria. For data collection, an evaluation form prepared by the researchers was used, an analog manovacuometer to measure the strength of the respiratory muscles by means of PImax and PEmax and a Peak-Flow to measure the peak expiratory flow. Results: most participants $(n=12)$

\footnotetext{
${ }^{1}$ Centro Universitário UniFacid, Teresina, PI, Brasil. Fisioterapeuta e Pós-graduanda em Fisioterapia TraumatoOrtopédica e Esportiva - Teresina, PI, Brasil.

${ }^{2}$ Universidade do Vale do Paraíba - São José dos Campos, SP, Brasil. Mestrando em Engenharia Biomédica pelo Instituto de Pesquisa e Desenvolvimento da Universidade do Vale do Paraíba - São José dos Campos, SP, Brasil.

${ }^{3}$ Centro Universitário UniFacid, Teresina, PI, Brasil. Graduada em Enfermagem pelo Centro Universitário UniFacid, Teresina, PI, Brasil.

${ }^{4}$ Centro Universitário UniFacid, Teresina, PI, Brasil. Doutora em Engenharia Biomédica pelo Instituto de Pesquisa e Desenvolvimento da Universidade do Vale do Paraíba e Docente do Centro Universitário UniFacid, Teresina, PI, Brasil.
} 
$66.7 \%$ were male and $(n=6) 33.3 \%$ female. Of the $(n=10) 55.6 \%$ smokers $(n=6) 60 \%$ had started smoking before the age of 18 . According to the nicotine dependence scale, $30 \%$ of the group had a high degree $(n=3)$. There was a statistically significant difference between average height among young people with level 1 in relation to those with level 2 of nicotine dependence (1.76 versus 1.67; $p=0.01$ ). Conclusion: therefore, the habit has deleterious effects, developing chemical dependency and altering the respiratory mechanics of young adults.

DESCRIPTORS: Young adults; Smoking; Respiratory mechanics; Physiotherapy.

RESUMEN: Objetivo: evaluar los efectos del tabaquismo en la mecánica respiratoria en adultos jóvenes. Métodos: este estudio está diseñado como una investigación de campo, con un enfoque cuantitativo, descriptivo y comparativo. La muestra del estudio consistió en 18 participantes, elegidos según criterios preestablecidos. Para la recolección de datos, se utilizó un formulario de evaluación preparado por los investigadores, un manovacuómetro analógico para medir la fuerza de los músculos respiratorios por medio de PImax y PEmax y un Peak-Flow para medir el flujo espiratorio máximo. Resultados: la mayoría de los participantes $(n=12) 66.7 \%$ eran hombres y $(n$ =6) $33.3 \%$ mujeres. Del $(n=10) 55.6 \%$ fumadores $(n=6) 60 \%$ había comenzado a fumar antes de los 18 años. Según la escala de dependencia a la nicotina, el 30\% del grupo tenía un alto grado $(\mathrm{n}=$ 3). Hubo una diferencia estadísticamente significativa entre la estatura promedio entre los jóvenes con nivel 1 en relación con aquellos con nivel 2 de dependencia a la nicotina (1.76 versus $1.67 ; \mathrm{p}=$ 0.01). Conclusión: por lo tanto, el hábito tiene efectos nocivos, desarrolla dependencia química y altera la mecánica respiratoria de los adultos jóvenes.

DESCRITORES: Adultos jovenes; Tabaquismo; Mecánica respiratória; Fisioterapia.

\section{INTRODUÇÃO}

Atualmente o tabagismo é considerado um problema de saúde pública, em razão do alto número de fumantes e da mortalidade decorrente das doenças relacionadas ao tabaco, sendo responsável por causar dependência química, física, tornando o seu tratamento mais difícil ${ }^{1}$.

A prática de fumar pode ser diferenciada conforme o gênero, idade, estado civil, ocupação, escolaridade e entre outras características $^{2}$. No Brasil a faixa etária para iniciação de consumos de produtos do tabaco começa entre 17 a 19 anos, sendo a proporção de mulheres que começam a fumar antes dos 15 anos superior à dos homens ${ }^{3}$.

O tabagismo pode limitar a capacidade aeróbica, ampliar a resistência ao fluxo aéreo e influenciar na função dos músculos respiratórios ${ }^{4}$. Um parâmetro clínico de escolha para avaliar as disfunções, é a mensuração da Força Muscular Respiratória (FMR), que se compõe na obtenção das pressões respiratórias geradas desde esforços inspiratórios e expiratórios máximos assim como a medição do pico de Fluxo Expiratório $(\mathrm{PFE})^{5}$. O hábito tabagista reduz o pico expiratório forçado, por meio de substâncias oriundas durante a queima do fumo, como por exemplo: alcatrão, nicotina e monóxido de carbono, sendo assim, desenvolvendo uma reação inflamatória que suscita em hipersecreção brônquica, contribuindo para o aparecimento da doença pulmonar obstrutiva crônica ${ }^{6}$.

A Pressão Inspiratória Máxima (PImax) corresponde ao índice de força da musculatura inspiratória, e a Pressão Expiratória Máxima (PEmax) ao índice de força dos músculos expiratórios ${ }^{7}$. As medidas PImax e PEmax são realizadas com a finalidade de verificar se existe fraqueza ou fadiga da musculatura respiratória que, por sua vez, podem causar diversas doenças do aparelho respiratório ou se apresentarem como consequência das condições clínicas adversas ${ }^{8}$.

Nesse caso pode-se observar que o tabagismo tem influência sobre a mecânica respiratória, desenvolvendo alterações de forma sistêmica, além de desenvolver 
dependência química, o que dificulta a cessação do hábito. Diante disso, este estudo objetiva avaliar os efeitos do tabagismo sobre a mecânica respiratória em adultos jovens.

\section{MATERIAIS E MÉTODOS}

Esse estudo é delineado como pesquisa de campo, de abordagem quantitativa, descritiva e de natureza comparativa. $\mathrm{O}$ universo da pesquisa foi uma instituição privada de ensino superior da cidade de Teresina-PI, sendo escolhida por ser referência e contemplar a amostra necessária ao estudo.

A coleta de dados ocorreu no período de março a abril de 2018, com uma amostra de 18 participantes, escolhidos de acordo com os critérios de inclusão e exclusão. Com critérios de inclusão foram: ambos os gêneros, com idade entre 20 a 40 anos, que afirmam nunca ter experimentado cigarro, como também tabagista que fumam ao menos uma unidade cigarro por dia, há pelo menos 1 ano. E sendo excluídos os que apresentaram diagnóstico de qualquer doença pulmonar ou cardíaco pré-existente, e que já tivessem fumado e abandonado o vício.

$\mathrm{O}$ instrumento de coleta de dados utilizado para os tabagistas e não tabagista foram: uma ficha de avaliação elaborada pelo autor contendo os seguintes itens: dados pessoais, peso, altura, índice de massa corpórea (IMC), início do hábito ou se nunca houve interesse para o consumo, motivação para cessar o hábito nos próximos seis meses, assim como o consumo em anos mensurados em maço de cigarros e uma tabela para constar os valores obtidos na avaliação de PImax, PEmax e PFE. A mensuração da força
Artigo Original dos músculos respiratórios foi obtida através da medida da PImax e da PEmax, utilizando o manovacuômetro analógico da marca GERAR ${ }^{\circledR}$ com escala de -150 a +150 $\mathrm{cmH} 2 \mathrm{O}$. As medições foram realizadas com os participantes na posição sentada confortavelmente, pés apoiados, mãos sobre as coxas, com clip nasal.

A PImax foi mensurada após uma expiração máxima, enquanto a PEmax foi obtida após uma inspiração máxima esperando-se estar próximo à capacidade pulmonar total. Os participantes realizaram de 3 a 5 manobras e obtendo 3 manobras aceitáveis. Durante a manobra para mensuração, foi solicitado sustentação por pelo menos 2 segundo e com valores próximos entre si $(\leq 10 \%)$, intercaladas com dois minutos de repouso. Entre as mensurações da PImax e da PEmax, houve um intervalo de cinco minutos. Dos três valores obtidos, tanto na PImax quanto na PEmax, apenas o maior valor reprodutível foi considerado. Os dados obtidos nos grupos tabagistas e não tabagista foram comparados e analisados.

Para a obtenção do PFE, foi utilizado Peak- Flow (Assess ${ }^{\circledR}$ - USA), graduado entre 0 - 880L/min. A avaliação foi realizada com o indivíduo sentado utilizando um clipe nasal, sendo assim, o participante realizava uma manobra expiratória forçada a partir da inspiração máxima, a nível de capacidade pulmonar total, sendo esta executada três vezes, com intervalo de um minuto, utilizando o maior valor alcançado. Os dados obtidos foram comparados aos valores preditos pela literatura, que leva em consideração a idade e a altura, proposto por Leiner et $\mathrm{al}^{9}$, separandose os gêneros, como mostra a tabela 1.

Tabela 1. Equações de Leiner et al. (1963).

\begin{tabular}{c}
\hline Homens \\
\hline PFE $(\mathrm{L} / \mathrm{min})=.[3,95-(0,015 \times$ idade em anos $)] \times$ altura em $\mathrm{cm}$ \\
MFE $(\mathrm{L} / \mathrm{min})=.[2,093-(0,0072 \times$ idade em anos $)] \times$ altura $\mathrm{em} \mathrm{cm}$ \\
\hline
\end{tabular}

Fonte: Leiner et al. (1963). 
Para mensurar o grau de dependência do tabaco, foi utilizado um questionário de tolerância de Fagerström, com objetivo de identificar a dependência a nicotínica. Conforme a soma dos pontos obtidos com as respostas referentes a seis perguntas, a dependência foi classificada em cinco graus: muito baixa (0 a 2), baixa ( 3 a 4$)$, média (5), elevada (6 a 7) e muito elevada (8 a 10) segundo a tabela de Fagerstrom ${ }^{10}$. Para o ponto de corte entre fumantes com baixa e elevada dependência do tabaco, foi adotada a mediana, com valor seis. Assim, os fumantes que somaram valor maior igual a seis pontos (dependência, elevada a muito elevada) foram considerados como: elevada dependência a nicotina, e aqueles com menos de seis pontos (dependência baixa a média) sendo considerados: baixa dependência.

A coleta de dados teve início após a assinatura do Termo de Consentimento Livre e Esclarecido. Além disso, o estudo foi previamente submetido à análise do Comitê de Ética em Pesquisa do Centro Universitário Unifacid, e aprovado com o número do parecer: 2.360.457 e do CAAE: 76696417.1.0000.5211, levando em consideração a importância da verificação das diretrizes e normas de pesquisas científicas em seres humanos, conforme preconiza a Resolução 466/2012 do Conselho Nacional de Saúde ${ }^{11}$.
Artigo Original

Os dados dos questionários foram tabulados através do programa Microsoft Office Excel versão 10. Posteriormente, a análise estatística foi realizada utilizando-se o Programa Statistical Package for the Social Sciences - SPSS, versão 17.0 e BioEstat 5.0.

Utilizou-se o teste $t$ de Student para análise comparativa das médias de variáveis do perfil da amostra segundo hábito do tabagismo e nível de dependência de nicotina e na comparação entre a performance da mecânica respiratória segundo a ocorrência ou não de tabagismo e de acordo com grau de dependência. Para todos os testes, considerouse como significativo $\mathrm{p}<0,05$. Os resultados foram organizados em gráficos e tabelas, nas quais foram descritos na forma de frequência, porcentagem e desvio padrão.

\section{RESULTADOS}

No presente estudo foram incluídos um total de 18 adultos jovens, sendo a maioria $(n=12) \quad 66,7 \%$ do gênero masculino e $(n=6)$ $33,3 \%$ do gênero feminino. Dos 55,6\% ( $\mathrm{n}=10)$ tabagistas $(60 \%, n=6)$ haviam iniciado fumo antes dos 18 anos. Segundo a escala de dependência de nicotina $30 \%$ do grupo apresentou grau elevado $(\mathrm{n}=3)$, conforme a tabela 2. 
Tabela 2. Perfil dos adultos jovens incluídos $(n=18)$ em estudo sobre influência do tabagismo na mecânica respiratória, em Teresina, PI.

\begin{tabular}{|c|c|c|c|}
\hline Variável & Categoria & $\mathbf{N}$ & $(\%)$ \\
\hline \multirow{3}{*}{ Sexo } & Feminino & 6 & 33,3 \\
\hline & Masculino & 12 & 66,7 \\
\hline & TOTAL & 18 & 100 \\
\hline \multirow{3}{*}{ Tabagista } & Sim & 10 & 55,6 \\
\hline & Não & 8 & 44,4 \\
\hline & TOTAL & 18 & 100 \\
\hline \multirow{3}{*}{ Curiosidade em relação ao fumo } & Sim & 3 & 37,5 \\
\hline & Não & 5 & 62,5 \\
\hline & TOTAL & 8 & 100 \\
\hline \multirow{3}{*}{ Início tabagismo (anos) } & $<18$ anos & 6 & 60,0 \\
\hline & $\geq 18$ anos & 4 & 40,0 \\
\hline & TOTAL & 10 & 100 \\
\hline \multirow{6}{*}{ Grau de dependência } & Muito baixo & 3 & 30,0 \\
\hline & Baixo & 2 & 20,0 \\
\hline & Médio & 2 & 20,0 \\
\hline & Elevado & 3 & 30,0 \\
\hline & Muito elevado & 0 & 0 \\
\hline & TOTAL & 10 & 100 \\
\hline \multirow{4}{*}{ Carga tabágica } & Até 2 anos & 2 & 20,0 \\
\hline & Entre 2 e 5 anos & 4 & 40,0 \\
\hline & Maior que 5 anos & 4 & 40,0 \\
\hline & TOTAL & 10 & 100 \\
\hline \multirow[t]{3}{*}{ Motivado a deixar o hábito } & Sim & 4 & 40,0 \\
\hline & Não & 6 & 60,0 \\
\hline & TOTAL & 10 & 100 \\
\hline
\end{tabular}

*participantes tabagistas. Fonte do estudo (2020).

Não houve diferença estatisticamente significativa entre as medias de idade $(\mathrm{p}=0,474)$, peso $(\mathrm{p}=0,494)$, altura $(\mathrm{p}=0,07)$ e índice de massa corporal $(\mathrm{p}=0,635)$ entre $\mathrm{o}$ grupo de jovens adultos não tabagistas e o de tabagistas. Do mesmo modo não houve diferença entre as médias de idade $(\mathrm{p}=0,406)$, peso $(p=0,578)$ e IMC $(p=0,485)$ entre os grupos segundo nível de dependência de nicotina. Tais resultados evidenciam homogeneidade da amostra estudada, diminuindo viés em relação a tais variáveis de acordo com os dados apresentados na tabela 03 .

Ainda, conforme a tabela 03, houve diferença estatisticamente significativa entre média de altura entre jovens com nível $1 \mathrm{em}$ relação aqueles com nível 2 de dependência de nicotina $(1,76$ versus 1,$67 ; \mathrm{p}=0,01)$, entretanto, a altura não apresenta correlação com grau de dependência a nicotina.

Tabela 3. Comparação de variáveis do perfil da amostra segundo hábito do tabagismo e nível de dependência de nicotina, Teresina, PI.

\begin{tabular}{|c|c|c|c|c|c|c|}
\hline \multirow[t]{2}{*}{ Variável } & \multicolumn{2}{|c|}{ TABAGISTA } & \multirow[t]{2}{*}{$p$ valor } & \multicolumn{2}{|c|}{ DEPENDÊNCIA } & \multirow[t]{2}{*}{$p$ valor } \\
\hline & SIM & NÃO & & NIVEL 1 & NIVEL 2 & \\
\hline Idade (anos) & 24,8 & 23,2 & 0,474 & 23,0 & 26,6 & 0,406 \\
\hline Peso $(\mathrm{Kg})$ & 72,2 & 69,0 & 0,494 & 74,0 & 70,4 & 0,576 \\
\hline Altura (m) & 1,72 & 1,65 & 0,07 & 1,76 & 1,67 & $0,01 *$ \\
\hline IMC (kg/m²) & 24,4 & 25,1 & 0,635 & 23,7 & 25,1 & 0,485 \\
\hline
\end{tabular}

Fonte do estudo (2020). Legenda: Nível 1 - muito baixa a média; Nível 2 - elevada e muito elevada. Valores expressos na forma de média. $\left.{ }^{*}\right)$ p valor estatisticamente significativo para teste t de Student. 
Não houve diferença estatisticamente significativa entre o grupo tabagista e não tabagista em relação a pressão inspiratória máxima $(\mathrm{p}=0,283)$, pressão expiratória máxima $(\mathrm{p}=0,572)$, pico de fluxo expiratório
Artigo Original $(\mathrm{p}=0,399)$ e razão entre pico de fluxo expiratório encontrado e predito $(\mathrm{p}=0,317)$, embora para todas essas variáveis, as médias tenham sido maiores no grupo não tabagista, segundo a tabela 04 .

Tabela 4. Comparação da performance da mecânica respiratória entre jovens adultos segundo ocorrência ou não de tabagismo, em Teresina, PI.

\begin{tabular}{cccc}
\hline Variável & \multicolumn{2}{c}{ TABAGISTA } & \multirow{2}{*}{ p valor } \\
\cline { 2 - 3 } & SIM & NÃO & 0,283 \\
\hline PI máxima & $-107,0 \pm 32,3$ & $-123,7 \pm 31,1$ & 0,572 \\
\hline PE máxima & $100,0 \pm 21,1$ & $105,6 \pm 19,8$ & 0,399 \\
\hline PFE & $710,0 \pm 169,9$ & $776,0 \pm 150,0$ & 0,317
\end{tabular}

Legenda: PI máxima - pressão inspiratória máxima; PE máxima - pressão expiratória máxima; PFE - pico de fluxo expiratório; PFE predito - pico de fluxo expiratório predito. Valores expressos na forma de média \pm desvio padrão. $p$ valor para teste $\mathrm{t}$ de Student.

Quando comparados segundo nível de dependência, não foi verificado diferença estatisticamente significativa entre os grupos em relação a pressão inspiratória máxima $(\mathrm{p}=0,789)$, pressão expiratória máxima $(\mathrm{p}=0,579)$, pico de fluxo expiratório $(\mathrm{p}=0,558)$ e razão entre pico de fluxo expiratório encontrado e predito $(\mathrm{p}=0,08)$, tabela 05 .

Tabela 5. Comparação da performance da mecânica respiratória segundo o nível de nicotina, em Teresina, PI.

\begin{tabular}{cccc}
\hline Variável & \multicolumn{2}{c}{ GRAU DEPENDÊNCIA } & \multirow{2}{*}{ p valor } \\
\cline { 2 - 3 } & NÍVEL 1 & NIVEL 2 & 0,789 \\
\hline PI máximo & $-104,0 \pm 42,8$ & $-110,0 \pm 22,4$ & 0,579 \\
\hline PE máximo & $104,0 \pm 11,4$ & $96,0 \pm 28,8$ & 0,558 \\
\hline PFE & $744,0 \pm 214,3$ & $676,0 \pm 127,0$ & 0,08 \\
\hline
\end{tabular}

Legenda: PI máxima - pressão inspiratória máxima; PE máxima - pressão expiratória máxima; PFE - pico de fluxo expiratório; PFE predito - pico de fluxo expiratório predito. Nível 1 - muito baixa a média; Nível 2 - elevada a muito elevada. Valores expressos na forma de média \pm desvio padrão. $p$ valor para teste $t$ de Student.

\section{DISCUSSÃO}

Nas características tabagísticas da amostra deste estudo, houve um maior número de fumantes no gênero masculino (70\%), quando comparado com o feminino (30\%), o que vai de acordo com a pesquisa realizada pela Vigilância de Fatores de Risco e Proteção para Doenças Crônicas por Inquérito Telefônico ${ }^{12}$, apontando que o percentual total de fumantes com 18 anos ou mais no Brasil é de $10,2 \%$, sendo $12,7 \%$ entre homens e $8,0 \%$ entre mulheres.

Um aspecto preocupante deste estudo refere-se ao início precoce do hábito de fumar, uma vez que mais da metade dos entrevistados tinham 23 anos ou menos. De acordo com a Pesquisa Nacional de Saúde do
Escolar $^{13}$, o indicador de experimentação de cigarro para os meninos $(19,4 \%)$ foi superior quando comparado às meninas $(17,4 \%)$, sendo a idade média de experimentação de tabaco entre jovens brasileiros é de 16 anos de idade, tanto para meninos quanto para meninas.

Para o nível de dependência à nicotina, analisado através do teste de Fagerstrom ${ }^{10}$, pôde-se constatar no presente estudo que a maioria dos participantes apresentou baixo índice de dependência que pode estar ligado ao fato de se enquadrarem em uma faixa etária jovem, com uma baixa carga tabágica, considerado importante para resultar em prejuízos respiratórios. No estudo de Melo et al. ${ }^{14}$ realizado com universitários de 18 a 23 anos, apresentou o grau de dependência nicotínica muito baixo, pelo fato de serem 
fumantes que se enquadram em uma faixa etária jovem, com consumo tabagístico concentrado abaixo de 10 anos-maço.

Com base no estudo de Nunes e Castro $^{15}$ o grau de dependência nicotínica aumenta progressivamente em faixas etárias mais avançadas, o que pode justificar o baixo índice de dependência à nicotina dos participantes da pesquisa. No entanto, sabe-se que $\mathrm{o}$ tabaco provoca tolerância $\mathrm{e}$ dependência, de modo progressivo, crônico e recorrente.

Por outro lado, os dados deste estudo evidenciam que $60 \%$ dos participantes não estão motivados a parar de fumar, sendo esta vontade um passo considerado importante para o sucesso do tratamento. Os fumantes que apresentam elevado grau de dependência, maior é a ocorrência e a gravidade da síndrome da abstinência o que o torna mais complicado manter a abstinência em longo prazo, necessitando assim além de abordagem cognitivo-comportamental, uma terapia mais intensa, para lograr êxito no abandono ${ }^{1}$.

Concordando com o estudo de Batista et al. $^{16}$ no qual considera a relação do tabagismo e o peso corporal, tornando o hábito favorável a um crescente aumento de acúmulo de gordura visceral e a resistência à insulina, resultando no aumento do risco de síndrome metabólica e diabetes tipo 2.

No estudo de Freitas, Araujo e Alves ${ }^{17}$, realizado com 120 idosos, verificou que existe alteração das medidas da FMR em idosos tabagistas quando comparados aos não tabagistas, principalmente na PImáx, a qual apresentou uma significante redução de seus valores no Grupo Tabagista $(\mathrm{p}<0,01)$. Entretanto, a PEmáx, apesar de não demonstrar significância estatística, houve uma discreta redução em seus valores quando comparado aos não fumantes.

Melo et $\mathrm{al}^{14}$, verificou a PFE em fumantes passivos e ativos de baixa dependência a nicotina, observou diferença estatisticamente significante no valor da PFE quando comparado o grupo de fumantes ativos e passivos, sendo identificada a diminuição da PFE em fumantes passivos.

Em um outro estudo, no qual avaliou a força muscular em mulheres fumantes, com idade de 18 a 40 anos e com grau de
Artigo Original

dependência a nicotina moderada, verificouse que os valores da PImax e PEmax obtidos foram menores que os previstos, constando que quanto maior o tempo de fumo maiores as alterações, afirmando também que a idade colabora para a fraqueza destes músculos, favorecendo o surgimento de problemas respiratórios ${ }^{18}$.

Dallosto et $\mathrm{al}^{19}$. realizou um estudo com amostra de 32 jovens universitários tabagista, com média de idade 22 anos e tempo médio de anos de hábito tabágico 5 anos, destes $87,5 \%$ mostraram baixa dependência a nicotina. Ao analisar os valores de PFE obtido com o previsto, observou uma redução significativa $(\mathrm{p}<0,001)$, pelo fato de os jovens da amostra apresentarem sintomas respiratórios. Contrapondo aos dados deste estudo, no qual os participantes tabagistas não apresentaram alterações da mecânica respiratória, consequentemente sem sintomas, podendo ser justificado pelo fato de serem jovens e com pouco tempo de consumo.

\section{CONCLUSÃO}

No que se refere ao perfil da amostra, foi constituído em sua maioria pelo sexo masculino, que iniciaram o tabagismo ainda na adolescência, apresentando baixa dependência e a não motivação para deixar o hábito. Nas variáveis de PImax, Pemax e PFE que não houve significância estatística entre o grupo tabagista e não tabagista, embora que para todas essas apresentarem as médias maiores no grupo não tabagista.

Diante do estudo, observa-se que o hábito apresenta efeitos deletérios, desenvolvendo uma dependência química e pode implicar na alteração da mecânica respiratória de adultos jovens.

Espera-se que outros estudos com amostra maior e com uma população de idade mais avançada em relação ao consumo do cigarro, possam mostrar alterações da mecânica respiratória e sua repercussão. Visando ajudar a desenvolver programas de atenção aos jovens para prevenção de início precoce do hábito tabagista, tendo em vista a redução das alterações provocadas e doenças 
correlacionadas, servindo também como fonte de pesquisa para outros estudos.

\section{REFERÊNCIAS}

1. Rossaneis MA, Machado RCBR. Cessação do tabagismo em pacientes assistidos em um ambulatório de tratamento de dependência do tabaco. Ciência, Cuidado e Saúde, 10(2), 2011, 306-313. [acesso em 2020 Jul 06]. Disponível em: https://doi.org/10.4025/cienccuidsaude.v10i2. 15688.

2. Meier DAP, Vannuchi MTO, Secco IAO. Abandono do tratamento do tabagismo em programa de município do norte do Paraná. Espaço para Saúde, 13(1), 2011. 3544. [acesso em 2020 Jul 21]. Disponível em: http://dx.doi.org/10.22421/1517-

7130.2011v13n1p35.

3. Kock SK, Barros HF. Efeitos do tabagismo na funcionalidade pulmonar. Journal of Health \& Biological Sciences 2014, 2(4), 176-181. [acesso em 2020 Jul 21]. Disponível em: http://dx.doi.org/10.12662/2317-

3076jhbs.v2i4.114.p176-181.2014.

4. Zanoni CT, Rodrigues CMC. Mariano D, Suzan ABBM, Boaventura LC, Galvão F. Efeitos do treinamento muscular inspiratório em universitários tabagistas e não tabagistas. Fisioterapia e Pesquisa 2012, 19(2), 147-152.

5. Pereira NH, Fernandes PM, Santos RNLC, Carvalho CPGP, Soares MEDS, Santos ACC. Comparação dos valores obtidos e previstos das pressões respiratórias máximas em adultos jovens. Ciência, Cuidado e Saúde 2015, 14(1), 955-961.

6. Silva JA, Fonseca MR, Melo MAV, Melo PM. O peak flow expiratório em mulheres fumantes e não fumantes e suas medidas de confiabilidade. Assobrafir Ciência 2015, 6(1), 41-48.

7. Reis JSD, Dantas MS, Silva CBD, Valverde L, Landeiro RBR. Caracterização da
Artigo Original

força muscular respiratória e da capacidade funcional de pacientes internados em uma enfermaria. Revista Fisioterapia \& Saúde Funcional, Fortaleza, 2012; 1(2):3-9, jul./dez.

8. Viana DA, Silva LMA, Rodrigues LR, Abrahão DPS, Gomide LB. Força Muscular Respiratória de Universitários Saudáveis. Revista Brasileira de Ciências da Saúde 2015, 31-34.

9. Leiner GC, Abramowitz S, Small MJ, Stenby VB, Lewis WA. Expiratory peak flow rate. standard values for normal subjects. use as a clinical test of ventilatory function. American Review of Respiratory Disease. 1963; 88:644-651.

doi:10.1164/arrd.1963.88.5.644.

10. Biblioteca Virtual em Saúde da Atenção Primária à Saúde. Teste de fagerström. [acesso em 2020 Jul 06]. Disponível em: https://aps.bvs.br/apps/calculadoras/?page $=12$

11. Ministério da Saúde (BR), Conselho Nacional de Saúde, Comissão Nacional de Ética em Pesquisa. Resolução No 466 de 12 de dezembro de 2012: diretrizes e normas regulamentadoras de pesquisa envolvendo seres humanos. Brasília: MS; 2012.

12. Brasil. Ministério da Saúde. Vigitel Brasil 2016: vigilância de fatores de risco e proteção para doenças crônicas por inquérito telefônico: estimativas sobre frequência e distribuição sociodemográfica de fatores de risco e proteção para doenças crônicas nas capitais dos 26 estados brasileiros e no Distrito Federal em 2016. Brasília: Ministério da Saúde, 2017.

13. Instituto Brasileiro de Geografia e Estatística. Pesquisa Nacional de Saúde do Escolar 2015. Rio de Janeiro: Coordenação de População e Indicadores Sociais, 2016.

14. Melo I, Cavalcante R, Magliano F, Duarte B, Oliveira AS. Análise do grau de obstrução de vias aéreas entre fumantes ativos e passivos acadêmicos da faculdade asces. 
Revista Inspirar Movimento \& Saúde 2017,

12(1).

15. Nunes SOB, Castro MRPD. Tabagismo: abordagem, prevenção e tratamento. $\quad$ EDUEL 2010. https://doi.org/10.7476/9788572166751.

16. Batista ES, Nascimento Campos T, Valente FX, Priore SE, Franceschini SDCC, Sabarense CM, Peluzio MDCG. Impacto do tabagismo e álcool sobre a composição corporal de jovens. Revista Brasileira de Cancerologia 2011, 57(3), 355-363.

17. Freitas ERFS, Araujo CCLS, Alves KS. Influência do tabagismo na força muscular respiratória em idosos. Revista Fisioterapia e Pesquisa, v. 19, n. 4, p. 326331, 2012.

18. Ruas G, Couto V, Pegorari MS, Ohara DG, Jamami LK, Jamami M. Avaliação da força muscular respiratória em mulheres fumantes. Saúde Coletiva 2013, 10(60), 1317.

19. Dallosto APZ, Zanchetta L, Mortari DM, Rockenbach CWF, Leguisamo CP. Grau de dependência nicotínica e valores espirométricos em acadêmicos tabagistas. Conscientia e Saúde 2009, 8(4), 587-592.

Recebido em: 07.07.2020. Aprovado em: 23.07.2020. 